\title{
Introduction - Des espaces du droit
}

Introduction - Law Space

Jacques Lévy

\section{(2) OpenEdition}

\section{Journals}

Édition électronique

URL : http://journals.openedition.org/rge/8010

DOI : 10.4000/rge.8010

ISSN : 2108-6478

\section{Éditeur}

Association des géographes de l'Est

\section{Référence électronique}

Jacques Lévy, «Introduction - Des espaces du droit », Revue Géographique de l'Est [En ligne], vol. 58 / 1-2 | 2018, mis en ligne le 25 avril 2019, consulté le 08 septembre 2020. URL : http://

journals.openedition.org/rge/8010; DOI : https://doi.org/10.4000/rge.8010

Ce document a été généré automatiquement le 8 septembre 2020

Tous droits réservés 


\title{
Introduction - Des espaces du droit
}

\author{
Introduction - Law Space
}

Jacques Lévy

1 Le droit est une ingénierie, une technologie sociale, un ensemble de discours et d'actions qui en découlent, un dispositif rendant possible un ordre politique et des actions publiques légitimes. En raison de son exhaustivité - rien n'échappe au droit -, c'est aussi un environnement de nature sociétale, modifiable, comme tous les environnements, qui interagit avec d'autres environnements sociétaux, tels que les espaces.

2 L'intelligence du social comprend une multitude de connaissances et de compétences. Le droit ne se situe pas au même endroit de cette constellation que la géographie. Ce serait plutôt la science politique qui constituerait l'homologue de la géographie, et l'urbanisme, celui du droit. Il faut garder en mémoire ce décalage et s'assurer de bien maîtriser des néologismes tels que "géolégal» ou, tout simplement le «et» de l'expression « droit et géographie ».

3 Suivant Hans Kelsen, on peut tout à fait accepter l'idée d'une Grundnorm, c'est-à-dire qu'il existe de l'amont à l'aval une cohérence de l'édifice juridique. Cela donne au droit une puissante dimension autoréférentielle, qui fait partie des spécificités de cet environnement. Cela n'empêche pas mais organise la porosité entre le droit et le reste $\mathrm{du}$ monde social. La question est alors : à quels niveaux le réel spatial interagit-il avec l'édifice juridique?

4 Pour répondre à cette question, on doit prendre en compte une dissymétrie entre droit et espace. En effet, la juridicité est produite dans des conditions restrictives (un individu ne produit pas de droit) tandis que chaque acteur, même le plus modeste, produit ses spatialités et contribue directement à la dynamique des espaces. En compagnie de l'éthique et du politique, le droit appartient à un ensemble de productions culturelles bien délimité « affectif-objectif » tandis que l'espace est présent dans les quatre cases du tableau (figure 1) tant au subjectif qu'à l'objectif, tant à l'affectif qu'au cognitif. 
Figure 1. Le droit au sein des productions culturelles.

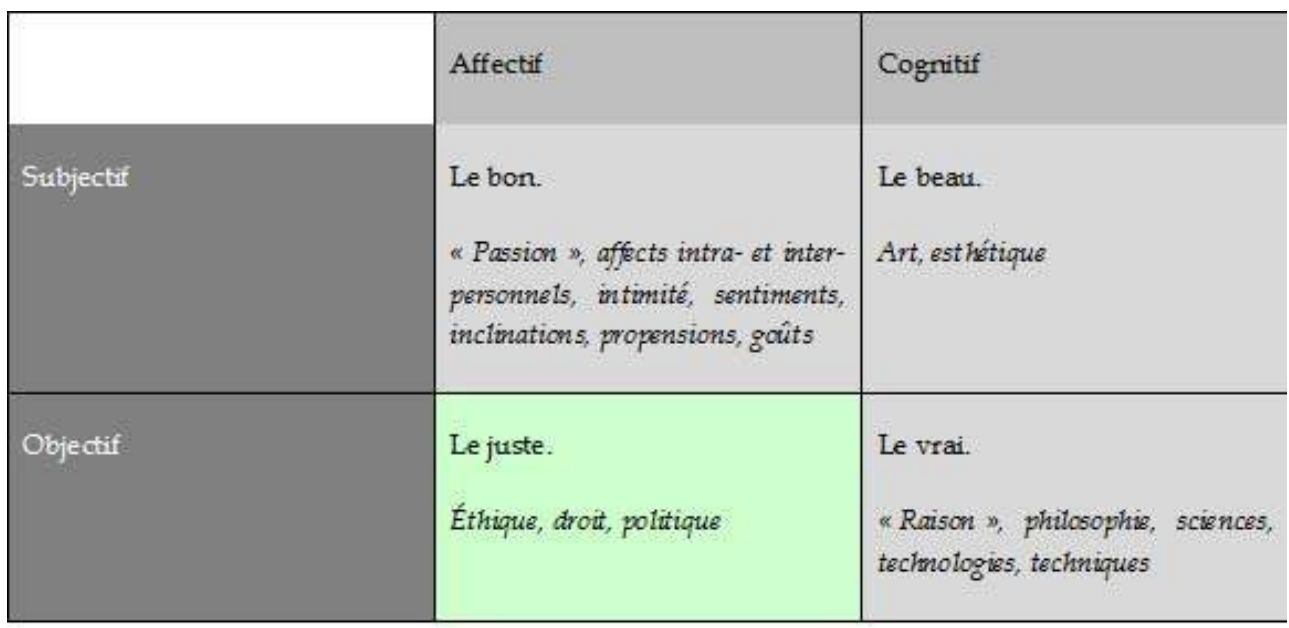

5 Cette dissymétrie s'affaiblit partiellement si l'on considère que la sphère politique, y compris la justice (judiciaire), n'a pas le privilège de la nomogenèse car la coutume (droit non écrit), la jurisprudence (droit des juges), la convergence juridique spontanée entre États ou encore les normes formalisées (soft law) sont aussi des configurations juridiques. Cette précision a son importance car elle permet de comprendre que l'existence d'un État stricto sensu ne constitue pas une condition exclusive pour la production d'un droit effectif. Il existe des réalités hybrides comme l'Union européenne ou les « organisations internationales » et des objets dérivés, comme les traités ou les accords de portée mondiale (tels que le Protocole de Kyoto ou l'Accord de Paris/Cop21 ou encore le projet d'harmonisation fiscale BEPS de l'OCDE) qui participent de la constitution des environnements juridiques.

$6 \mathrm{Vu}$ sous cet angle, le droit n'est donc pas seulement poreux, il est aussi liquide, il se répand et se mélange à ce qui n'est pas le droit, tout en restant, en gros, dans la même case du tableau présenté plus haut. Il semble évident qu'on ne puisse pas penser la science, l'art ou l'amour à partir du seul droit et ce cloisonnement relatif constitue un garde-fou essentiel pour une société ouverte, comme l'a montré Michael Walzer dans son livre Sphères de justice (1997 [1983]).

7 En somme, la rencontre entre juridicité et géographicité procède de l'intersection entre un univers relativement isolable et à forte prédominance sociétale et un autre qui associe la socialité (par la spatialité) et la société (par les espaces). Le dossier Géographies légales explore plusieurs aspects de cette intersection.

\section{Géographies du droit : l'espace de la république}

Le 20 mars 2019, Zuzana Caputova a été élue présidente de la Slovaquie sur un programme de lutte contre la corruption et les violences qu'elle engendre, inspiré par une association dans laquelle elle a milité, Via Iuris - «le chemin du droit ». À la même période, des centaines de milliers d'Algériens descendaient dans la rue pour réclamer l'instauration d'un véritable état de droit. Pendant les mêmes semaines, alors que les Gilets jaunes faisaient peu de cas, chaque samedi aux Champs-Élysées, du respect de la loi au nom d'une urgence sociale impérieuse, chaque dimanche à la République, les 
Algériens de Paris plaçaient en tête de leur exigences l'accès du pays, enfin, à l'état de droit.

9 On touche ici à la république au sens que lui donne Immanuel Kant (1971 [1795] ; 1991 [1795]. À la différence de la démocratie, pour laquelle le droit fonctionne comme une ingénierie qui rend concrètement viable une option (l'accès universel à la citoyenneté) qui n'est pas en elle-même de nature juridique, la république peut être lue comme une construction dont le droit est à la fois la substance (par la procédure et la transparence des décisions, par la séparation des pouvoirs, par la protection des individus de l'arbitraire) et la pierre angulaire, par sa force non réductible et non négociable, son intangibilité. C'est l'état de droit (rule of the law), souvent écrit, à tort, en français «État de droit ». En fait, cette expression n'évoque pas un État qui respecterait le droit, mais un environnement social où le droit prime sur la force.

10 Ce repère est d'autant plus utile que la république peut être pensée en tant que telle, sans la démocratie. Celle-ci ne fait pas à proprement parler du programme des Lumières tel qu'il apparaît chez Immanuel Kant ou Charles de Montesquieu, tandis que sur l'autre versant, plus ambivalent, représenté notamment par Jean-Jacques Rousseau, héritier de la tradition issue des courants du «droit naturel » et constituant un axe historique fort qui court de la Guerre des Paysans au communisme des XIX et XX siècles, la démocratie peut être brandie contre la république. Les totalitarismes se caractérisent précisément par la volonté d'utiliser les «masses» (le "prolétariat », le " peuple »), instituées comme communautés transcendant tout autre lien social, pour faire sauter les protections contre le despotisme que constitue le droit. Inversement, une fois installé au pouvoir, le nazisme a utilisé la « règle de droit » (Rechtsnorm), c'està-dire la légitimation par la loi, contre les principes de la république, faisant du droit un avatar monstrueux de sa propre histoire.

11 Ces constats vont à l'encontre des interprétations, dominantes dans la tradition marxiste, consistant à faire du droit un sous-produit superstructurel de rapports de force extérieurs. Dans la hiérarchie causale que proposait cette tradition, le matériel l'emportait sur l'immatériel et le concret sur l'abstrait. Étant, comme d'ailleurs le politique dans son ensemble, à la fois immatériel et abstrait, le droit était considéré comme un simple ajustement de ce qui se passait ailleurs, dans la sphère économique, où la tension entre "force productives » et "rapports de production » était censée rendre compte de chaque situation sociale. Le droit était vu comme un moyen d'encadrement et d'endoctrinement permettant de faire croire à la naturalité du système. Les révolutions se chargeraient de "remettre les pendules à l'heure " en adaptant le droit au nouvel ordre social. En réalité, le droit est une réalité digne d'intérêt en elle-même et c'est à ce titre que sa rencontre avec l'espace fait sens.

\section{Le droit comme environnement social}

12 Le fait que le droit porte aussi sur l'espace ne fait pas débat. On peut trouver dans des textes juridiques très anciens des règles consistant à différencier les lieux en raison d'un principe quelconque. Ce qui est nouveau, c'est la prise de conscience du rôle du droit dans l'agencement et la configuration du monde social.

13 Ainsi, Nadia Belaidi montre comment les objectifs d'une loi créent un cadre qui s'impose aux objets inclus dans ce cadre et les modifient, leur donnant un sens 
nouveau. Le droit fonctionne donc comme un environnement influençant les réalités qu'il englobe et, parmi ces réalités, il peut y avoir d'autres environnements, tels qu'un espace naturel protégé. Le monde social peut être défini comme un ensemble d'interactions entre acteurs, objets et environnements. Une société peut être vue comme un environnement particulièrement englobant puisqu'elle inclut, à son échelle, tous les autres, y compris les deux grandes familles d'environnements naturels, la Terre et les corps des humains. Par son omniprésence, le droit est un " équivalent général » qui non seulement socialise mais sociétalise tous les acteurs, tous les objets et tous les autres environnements que lui-même. Ce paysage juridique ("nomosphère », lawscape) n'est pas toujours aisément perceptible, nous dit Luke Bennett, qui invite les chercheurs comme les individus ordinaires à assumer le rôle d' " enquêteurs spatiaux " (spatial detectives) pour comprendre comment le formalisme normatif du droit contribue à organiser les lieux et les liens entre les lieux. Associant le droit et l'espace comme co-fabricants des lieux, Bennett indique un angle mort laissé en friche par les choix théoriques d'un ensemble constitué par la "géographie critique», la "géographie humaniste " et la "géographie culturelle", qui ont mis l'accent sur la nébuleuse auto-organisée de multiples subjectivités plutôt que sur le pouvoir organisateur des logiques sociétales, parmi lesquelles celle du droit. En lisant le roman Scarp (2012) de Nick Papadimitriou, un écrivain "psychogéographe", Bennett revalorise le droit comme une infrastructure sociale omniprésente mais peu visible qui code tous les objets, tous les moments et tous les emplacements dont le quotidien est fait. Le droit est ici, c'est aussi avec lui qu'on doit faire quand on fait avec l'espace. Autrement dit, nos spatialités ne peuvent ignorer l'espace juridique.

\section{Le droit comme ressource spatiale}

14 Il existe donc une histoire mais aussi une géographie de la place du droit dans le système politique. Qu'il y ait ou non présence du droit est en soi un événement géographique, ce qu'illustre l'analyse de Marie Mellac, sur la bifurcation, du droit foncier au Vietnam et au Cambodge d'un monopole étatique à la possibilité d'une propriété privée, moyennant diverses situations intermédiaires qui créent des stabilisations provisoires, par la loi, d'une mutation sociale majeure. Il ne s'agit pas seulement d'un élargissement des profils des propriétaires mais aussi de la notion même de propriété, qui hésite entre plusieurs régimes sémantiques et pratiques. Entre les indivisions communautaires des collectivités rurales non étatiques, le système féodal, qui distingue propriété et possession, l'approche étatiste du communisme, le protectionnisme souverainiste et la banalisation de la terre comme un bien parmi d'autres, de multiples combinaisons sont possibles. L'hybridation n'est pas toujours bien maitrisée ce qui entraîne des contradictions internes aux corpus juridique, d'autant que l'influence d'acteurs mondiaux impose parfois une improvisation dommageable à la cohérence.

Dans tous les cas de figure, la puissance organisatrice du droit sur l'ensemble des pratiques impliquant le sol est incontestable. C'est souvent le pouvoir central qui utilise le droit comme outil de légitimation, car ses acteurs disposent d'un avantage cognitif. En outre, la juridicisation constitue une rupture vis-à-vis des pratiques locales se situant dans des logiques communautaires non étatiques, comme on peut le voir dans beaucoup de situations d'achat de terres où les États concernés et des firmes 
transnationales concordent à affaiblir, au nom du droit, un monde rural réfractaire à la monétarisation du sol. L'imposition du droit participe donc activement de changement systémique dont il est à la fois une composante et un levier.

Lorsque le droit est bien installé au sein d'une société d'acteurs, il apparaît aussi comme une ressource pour ceux, individus ou collectifs, qui s'estiment victimes d'injustices et qui pensent pouvoir compter sur l'impartialité ou la bienveillance du juge pour annuler cette injustice. Les attributs propres de la sphère juridique jouent alors pleinement leur rôle en facilitant la production d'énoncés s'appliquant à toutes les situations comparables et, en conséquence, à diffuser dans tous les interstices de la société le même message. Cependant, dans cette production, les professionnels du droit, avocats et magistrats, sont conduits à chercher un ajustement entre la conception de l'espace qu'ils tirent des corpus juridiques et celles que proposent les parties à la procédure.

17 Comme le montre Marie Meténier à propos du cas britannique de la périurbanisation d'un espace autrefois agricole et désormais patrimonialisé en tant que paysage, les juges sont amenés à définir des principes pour fonder leurs décisions, en s'écartant des textes de manière à pouvoir arbitrer entre des légitimités multiples. Cet écart est plus aisé dans les pays anglophones, où le droit écrit est suffisamment restreint pour laisser de larges marges de manœuvre à l'interprétation. Aux États-Unis, en 1996, un juge fédéral a ainsi interdit au comté de Los Angeles d'étendre le réseau de métro tant qu'il n'aurait pas suffisamment investi dans celui des bus, en arguant du fait que les usagers des bus se trouvaient indûment désavantagés par le choix du métro. On pourrait considérer qu'il se substituait ainsi aux citoyens et à leurs représentants pour définir une politique publique de son cru. Cependant, même en Europe continentale, on observe de nombreux cas de jugements qui se fondent, pour dire le juste, sur des appréciations des modes d'habiter, sur des controverses scientifiques concernant le monde bio-physique ou sur des options esthétiques, en prenant inévitablement leur distance vis-à-vis des codes ou des jurisprudences qui évoquent peu, voire pas du tout, ces enjeux.

\section{Frontières légales, frontières du droit}

Ce n'est pas en soi une nouveauté pour le droit, dont le versant herméneutique est totalement imbriqué avec celui de l'énonciation de la norme. Ce qui est nouveau, c'est que cette liberté interprétative s'applique à des domaines où, si le droit est lacunaire, c'est pour une part parce qu'ils ne relèvent pas du droit. En matière d'espace, il existe de nombreuses configurations qui résultent dans des jeux sociaux à somme positive, sans antinomie entre les finalités des différents acteurs. Or, autant il est relativement facile d'identifier un vol puisqu'il s'agit de tracer le parcours d'un bien monétaire isolable qui est passé des mains du volé à celui du voleur, autant seule la société urbaine dans son ensemble peut juger si un projet d'urbanisme est cohérent, s'il est juste et s'il contribue au développement, toutes choses qui ne pourront peut-être être évaluées que longtemps après la mise en œuvre du projet. La différence entre évaluation et jugement permet de comprendre ce décalage. L'espace est profondément politique mais il est produit à chaque instant par une multitude d'acteurs dont la majorité appartient à la société civile et c'est justement pour cette raison que ces acteurs, maîtrisant de mieux en mieux la machinerie juridique, font de plus en plus appel au droit. 
19 Dans ce nouveau cadre, on perçoit bien comment le formalisme du droit entre en collision avec des espaces dont les échelles sont souvent enchevêtrées et les métriques, multiples. Les spatialités sont le fait d'acteurs disparates qui poursuivent des fins d'une grande diversité. Il ne s'agit pas ici de subtilités - l'histoire du droit et de la justice montre l'extraordinaire flexibilité du monde juridique. Il s'agit de complexité, et plus particulièrement de complexités pour lesquelles le droit, dans son principe, est mal équipé. On pourrait comparer ce genre de malaises à ce qui se passe lorsque, dans les affaires «familiales » ou de " mœurs », les juges se trouvent induits, parfois contre leur gré, à entrer dans des univers affectifs difficiles à explorer dans lesquels ils risquent de se perdre et de s'éloigner de leur mission. Dans toutes ces situations, le droit peut se trouver devoir jouer un rôle à contre-emploi car dans une société d'égalité et de liberté, il doit faire en sorte de laisser aux acteurs le maximum de place - et cette place comprend aussi la fabrique de l'espace. On touche là sans doute à une spécificité du rapport entre droit et géographie: le fait que ces deux familles d'environnements interagissent de manière croissante mais en étant de moins en moins superposables.

Dans un travail d'une grande précision, Marcela Iacub (2008) a décrit les longs débats de la sphère juridique européenne, du XIX ${ }^{e}$ siècle à aujourd'hui sur les limites entre espace public et espace privé. Ces deux familles d'espaces se sont inventées ensemble à cette époque, en se référant l'un à l'autre. Dans le partage, il est fortement question de métriques car il convient de déterminer si la vue de scènes interdites (par la fenêtre ou par le trou d'une serrure) est de même nature que la présence du voyeur à l'intérieur de l'appartement ou de la pièce où les choses se passent. La mission que s'assignait le droit, en particulier par la jurisprudence, consistait à dessiner une frontière entre deux configurations spatiales singulières. En effet, l'espace public a acquis un statut infrajuridique en ce qu'il se régule pour une bonne part par l'interaction informelle entre ceux qui le pratiquent. L'espace privé, de son côté, reprend l'héritage du respect accordé au communautarisme familial et fonctionne comme un hétérotope juridique : par défaut, la loi s'en désintéresse, en ne contrôlant pas en temps réel ce qui s'y passe, contrairement à l'espace public, quadrillé par les patrouilles de police et les caméras de surveillance. Dans les régimes totalitaires, ces deux types d'espaces perdent leur part d'autorégulation, mais de manière différenciée. En Iran, les autorités ne laissent ni un mètre-carré ni une seconde de liberté dans l'espace public, tandis que depuis les années 2000 , elles ont fini par se retirer, à contrecœur, de l'univers du logement, ce qui permet aux individus d'y exister comme tels en rencontrant qui ils veulent ou en regardant les chaînes grâce à leurs paraboles sans trop craindre les descentes de police. Les géographies du droit ont donc aussi pour enjeu la limitation, par la société civile, du champ de manœuvre d'un droit liberticide. Entre le public et le privé, entre la sphère de légitimité du droit et ses zones grises, la télécommunication numérique rebat les cartes.

\section{Quel espace pour le droit en deçà et au-delà de l'État ?}

L'une des difficultés dans la mise en cospatialité, c'est-à-dire dans la possibilité d'interactions mutuellement productives entre l'espace du droit et les autres espaces sociaux, tient au formalisme proprement géographique de la nomosphère. La montée en puissance d'États en Europe, puis dans le Monde dans la perspective westphalienne de l'exclusivité politique interne et de l'exclusivité géopolitique externe a fabriqué un 
monde juridiquement simple mais qui se trouve en conflit avec deux logiques majeures : celle qui met en cause la place de la violence dans la régulation des conflits entre sociétés et celle qui installe le politique à des échelles infra- et supranationale. En explorant les statuts des "pré-majeurs» en Europe, Rodolphe Dumouch montre comment le formalisme du droit se combine avec la topologie des frontières pour fabriquer des discontinuités se renforçant mutuellement et peinent à prendre en compte les trames de gris, les glissements et les gradients auxquels cette thématique devrait conduire.

Pour Carl Schmitt (2008 [1950]), juriste nazi, la « solution » est toute trouvée : il y a de l'espace en amont de toute loi et cet espace est celui que dessine la violence libérée du droit. Pour lui, le politique sera inévitablement écrasé par la géopolitique et il faut s'en accommoder. On peut cependant imaginer, symétriquement, un univers dans lequel la géopolitique serait surpassée par le politique. On peut ainsi dérouler le processus dans l'autre sens : la posture «idéaliste » en relations internationales consiste à traiter les relations intersociétales comme des relations politiques, avec le dessein d'éviter la guerre et à établir une méthode de pacification des conflits, incluant le recours à la violence légitime comme à l'intérieur d'une société où règne l'état de droit. Les relations inter-sociétales deviennent alors une composante particulière du politique, comme on peut l'observer dans les systèmes fédéraux, dans lesquels seul un niveau, le plus englobant en général, possède la compétence d'interface avec les autres sociétés (politique étrangère, défense). À l'intérieur de ce système interscalaire, les relations fédéral/régional/local sont de type politique et non géopolitique. Si on élargit la démarche en ne limitant pas le niveau supérieur, on aboutit à l'existence d'un cadre juridique englobant, donc d'un droit mondial.

Or ce droit n'est nullement le développement du droit international. Héritier du jus gentium (droit des gens, c'est-à-dire des peuples ou des nations, de groupes définis par un principe de filiation biologique) qui est un droit interstitiel dans une zone de non-droit, ce dernier s'installe dans le cadre westphalien comme les règles d'un wargame fondamentalement antinomiques avec un droit conçu comme formalisation de règles fondées sur des valeurs de la vie en société. La guerre en elle-même consiste à tuer des innocents, civils ou militaires, elle ne peut entrer dans une logique juridique. Si, au contraire, on est en présence d'une "guerre juste ", on entre dans le cadre d'un droit englobant et devient une opération de police pour empêcher des délinquants de nuire.

La construction depuis Solferino (1859) de normes humanitaires (soft law) puis, depuis Nuremberg (1945-46) de l'esquisse d'un droit mondial (global law), théorisé par Mario Bettati (1996) et Mireille Delmas-Marty (2004-2011), a fait plus que jurisprudence, confortée par la création de la Cour pénale internationale (CPI, 1998) et l'obligation de protéger mise en place par l'ONU en 2005. Il s'agit d'un droit intérieur, et de substance constitutionnelle puisqu'il opère une traduction juridique des valeurs fondatrices de la vie en société (droits de l'homme, droits civiques élémentaires, interdiction des crimes de guerre, du génocide et des crimes contre l'humanité).

Or est facile de montrer que le droit international et le droit mondial sont antinomiques, dessinant deux géographies, celles du système interétatique et celle de la société-Monde: pour s'en convaincre, il suffit de confronter la Charte des NationsUnies, qui garantit l'intangibilité de la souveraineté des États géopolitiques et la Déclaration universelle des droits de l'homme, à laquelle pourtant la première se réfère et qui place ses principes au-dessus de la souveraineté des États. Dans le cas du conflit 
du Kosovo (1999), la collision entre les deux types de droits a été manifeste puisqu'il n'était pas possible de défendre les droits de l'homme sans mettre en cause la souveraineté de l'État serbe.

\section{La mondialisation interpelle le droit}

Il faut aussi noter qu'un droit privé mondial est en train d'émerger à partir des jurisprudences qui compensent les lacunes et les conflits de compétence des droits nationaux. Il s'agit de répondre à des questions à la fois simples et redoutables : quel tribunal est compétent? quel droit est applicable? à quelles conditions peut-on appliquer un droit en dehors de ses frontières? En apparence, chaque pays décide de son propre degré et de sa propre méthode d'internationalisation, ce qui crée de nouveaux conflits, notamment en raison des exceptions justifiées par l'« ordre public " qui, en pratique, privilégient les principes fondateurs du droit pour résoudre les antinomies pratiques. Cela favorise un shopping juridique des justiciables à la recherche du traitement le plus avantageux, et c'est seulement dans les ensembles à tendance fédérale, comme l'Union européenne, que les ambiguïtés sont peu à peu réduites, comme on le voit avec la Convention de Lugano de 2007 (Union européenne, Norvège, Islande et Suisse).

Cependant, on voit aussi apparaître des logiques de droit privé à l'échelle mondiale, par exemple à propos de l'identité sexuelle ou du droit de la filiation. Un enfant né grâce à une gestation pour autrui (GPA) dans un pays où elle est autorisée et qui rejoint le pays dont ses parents ont la nationalité, où elle ne l'est pas, peut-il être privé de parents ? En 2014, la Cour européenne des droits de l'homme a imposé à l'État français de reconnaître la filiation d'un enfant né par GPA aux États-Unis, car il y a une valeur supérieure à la prohibition française de la GPA : le droit supérieur des enfants (identité, droit à une vie privée et notamment droit d'avoir des parents, absence de menace pour sa vie et ses droits).

On observe donc une construction juridique transnationale, fondée sur des valeurs communes comme les droits des enfants, eux-mêmes portés par le très ancien principe de dissociation entre procréation biologique et statut de parent et par un principe éthico-juridique fondamental selon lequel il ne saurait y avoir de crime sans victime. En l'occurrence, la seule victime, c'est le droit national puisque tous les acteurs sont adultes et consentants. La simple existence d'un espace mondial qui déstabilise les apories des droits nationaux stimule une dynamique juridique dont les changements d'échelle ont logiquement pour effet d'opposer des valeurs à vocation universelle à des particularismes non conformes à ces valeurs. En observant ce qui émerge, on mesure toute la différence avec le droit international, qui n'est qu'un ensemble d'arrangements visant à l'évitement ou à la résolution de conflits inutiles (comme la Cour internationale de justice de La Haye et d'autres tribunaux d'arbitrage) tandis que le droit international privé évolue sur la base d'une substance éthique de plus en plus consistante et de plus en plus unifiée.

On observe de multiples situations où le droit étatique classique est défié par des interspatialités (Lévy \& Lussault, 2013), c'est-à-dire des interactions entre espaces, à l'intérieur même des géographies du droit, qu'elles procèdent de la juxtaposition, de l'emboîtement ou de la superposition. Olivier Dubos (2001) a ainsi montré que le droit 
des juges n'est pas le droit des textes car même un texte international peut fort bien être interprété différemment dans des États différents.

Comme l'a analysé Boris Beaude (2012), l'Internet tend à devenir un lieu, mais un lieu réticulaire, dont les distances caractéristiques diminuent en fonction de la fluidité d'un espace fait de nœuds et des liens. Le numérique rend donc encore plus visible le problème général qu'un espace à dominante réticulaire pose au droit: ici, l'effet de ciseau est massif entre les lacunes du politique et la demande de cadre juridique. L'Internet encaisse et réfracte la collision entre un droit traditionnellement territorial, national et étatique et des pratiques préférentiellement réticulaires, mondiales et produites par une multitude d'acteurs. De ce conflit peuvent résulter la fin de l'Internet par renationalisation du droit tout autant que l'émergence d'un droit mondial, dont la mise en place suppose, au-delà d'ajustements techniques entre systèmes juridiques nationaux, un débat public mondial sur les valeurs communes fondant cette coalescence. Pour le moment, c'est un peu les deux (Beaude, 2014 ; Guillemot, 2014).

Tout cela signifie que l'observateur de la mondialisation du droit ne doit pas hésiter à changer de lunettes afin de détecter les mouvements pertinents en dehors des champs habituels, y compris, bien évidemment, dans les effets des mondes non juridiques sur le droit. C'est fondamentalement la mondialité du social en train de s'inventer qui permet de saisir les contradictions et les dynamiques de la mondialité du droit.

Comme souvent, si l'on accepte de prendre au sérieux les concepts et les langages d'un autre domaine, on rencontre non seulement des ressemblances inattendues et des terrains communs mais on fait aussi émerger des problèmes que, pris à part, ni l'une ni l'autre des disciplines n'avait été capable d'identifier. L'exploration des spatialités spécifiques du droit et les juridicités propres de l'espace ouvre sur des questions inédites telles que celles-ci : comment prend en compte la prééminence de réseaux mobiles dans des systèmes juridiques qui ont toujours considéré la territorialité et la fixité comme des cadres prééminents? peut-on utiliser le droit comme outil de construction d'espaces sociétaux là où le politique n'y est pas encore institué ? comment penser conjointement la mise en mouvement des environnements juridiques et des environnements spatiaux lorsque de nouveaux acteurs, les individus, deviennent la force instituante la plus dynamique de la société?

\section{BIBLIOGRAPHIE}

Beaude, Boris, 2012, Internet, changer l'espace, changer la société, Limoges, Fyp.

Beaude, Boris, 2014, Les fins d'Internet, Limoges, Fyp.

Bettati, Mario, 1996, Le droit d'ingérence, Paris, Odile Jacob.

Delmas-Marty, Mireille, 2004-2011, Les forces imaginantes du droit, Paris, Seuil, 4 vol. 
Dubos, Olivier, 2001, Les juridictions nationales, juge communautaire : contribution à l'étude des transformations de la fonction juridictionnelle dans les États membres de l'Union européenne, Paris, Dalloz.

Guillemot, Luc, 2014, Une fabrique de la société-Monde : Internet entre technique et politique mondiale, thèse de doctorat, Lausanne, ÉPFL.

Iacub, Marcela, 2008, Par le trou de la serrure : une histoire de la pudeur publique, $\mathrm{XIX}^{\mathrm{e}}$ et $\mathrm{XX}^{\mathrm{e}}$ siècle, Paris, Fayard.

Kant, Immanuel, 1971 [1795], Doctrine du droit, Paris, Vrin, 1971.

Kant, Immanuel, 1991 [1795], Projet de paix perpétuelle, Paris, GF-Flammarion.

Lévy, Jacques \& Lussault, Michel, 2013, Dictionnaire de la géographie et de l'espace des sociétés, Paris, Belin, nouvelle édition.

Schmitt, Carl, 2008 [1950], Le nomos de la terre, Paris, PUF.

\section{RÉSUMÉS}

Les rencontres entre l'espace et le droit sont complexes et prometteuses. Elles tirent parti de leur forte prégnance respective et aussi de leurs dissymétries : le droit fabrique un environnement omniprésent dans la vie sociale et son existence constitue en elle-même un enjeu mais il est ancré dans la sphère de l'éthique et du politique tandis que l'espace parce qu'il est produit par les spatialités de multiples acteurs intervenant dans de multiples registres et à de multiples échelles, est aussi un environnement mais dont la cospatialité avec celui du droit est à la fois fondamentale et problématique, dans un contexte où l'interaction entre les deux domaines ne fait que croître.

Encounters between space and law are complex and promising. They are nurtured by their dual pervasiveness but also by their asymmetry. Law generates a socially ubiquitous environment but remains anchored in the political-ethical sphere, as space is an environment, too, but it is produced by multi-realm, multi-scale, multi-actor spatialities. As a result, co-spatiality between space and law is simultaneously crucial and not to be taken for granted in a context where their relationships are undeniably flourishing.

\section{INDEX}

Mots-clés : droit, espace, spatialité, acteurs, environnement, cospatialité

Keywords : Law, Space, Spatiality, Actors, Environment, Co-spatiality

\section{AUTEUR}

\section{JACQUES LÉVY}

Professeur à l'École polytechnique fédérale de Lausanne et à l'Université de Reims ; Membre du rhizome Chôros, Université de Reims Champagne-Ardenne, UFR Lettres et Sciences Humaines, 57, rue Pierre Taittinger, 51100 REIMS 